\title{
La relación fuerza-velocidad para la optimización del entrenamiento y prevención de lesiones
}

The strength-velocity relationship for training optimization and injury prevention

Christian Rodolfo Morocho Ubidia ${ }^{1}$.

Recibido: 13-10-2020 / Revisado: 15-11-2020 /Aceptado: 10-12-2020/ Publicado: 02-012021

Abstract.

DOI: https://doi.org/10.33262/cienciadigital.v5i1.1462

Introduction. Strength and speed are determining abilities in the practice of different sports. Achieving the best ratio between the two is an important tool for optimization in sports training, as well as for the prevention of injuries. Objective. Reflect on the relationship between strength and speed and its importance for optimizing training and preventing injuries. Methodology. The research I consider a methodology is descriptive, with emphasis on the use of theoretical methods and documentary review. Results. The study identified 8 investigations as potential sources, of which 2 confirm the importance of strength training for injury prevention and the rest (6) emphasize the importance of strength and speed exercises to ensure training optimization sports. This made it possible to systematize the importance of the relationship between strength-speed capabilities as a viable tool to achieve optimization in sports training and in turn prevent the appearance of possible injuries. Conclusion. The high correspondence that is achieved when an adequate ratio is established between both capacities and the impact that this brings both in sports training and in injury prevention was confirmed.

Keywords: strength, speed, optimization, training, injuries

\section{Resumen.}

Introducción. La fuerza y la velocidad son capacidades determinantes en la práctica de los diferentes deportes. Lograr la mejor proporción entre ambas constituye una herramienta importante para la optimización en el entrenamiento deportivo, así como para la prevención de lesiones. Objetivo. Reflexionar sobre la relación entre la fuerza y la

\footnotetext{
${ }^{1}$ Universidad central del Ecuador Quito, Ecuador, crmorochou@uce.edu.ec ORCID: https://orcid.org/0000-0001-7876-5714
} 
velocidad y su importancia para la optimización del entrenamiento y la prevención de lesiones. Metodología. La investigación contemplo una metodología es descriptiva, con énfasis en el empleo de métodos teóricos y la revisión documental. Resultados. El estudio determinó 8 investigaciones como fuentes potenciales, de los cuales 2 confirman la importancia del entrenamiento de la fuerza para la prevención de lesiones y el resto (6), enfatizan en la importancia de los ejercicios de fuerza y velocidad para garantizar la optimización del entrenamiento deportivo. Esto permitió sistematizar la importancia de la relación entre las capacidades fuerza-velocidad como una herramienta viable para alcanzar la optimización en el entrenamiento deportivo y a su vez prevenir la aparición de posibles lesiones. Conclusión. Quedó constatada la alta correspondencia que se logra cuando se establece una adecuada proporción entre ambas capacidades y la repercusión que esto trae tanto en el entrenamiento deportivo como en la prevención de lesiones.

Palabras clave: fuerza, velocidad, optimización, entrenamiento, lesiones.

\section{Introducción.}

Buscar alternativas conducentes a la optimización en el entrenamiento deportivo que permitan no sólo alcanzar mejores resultados, sino también prevenir la aparición de posibles lesiones, sigue constituyendo un tema de gran preocupación para los investigadores, entrenadores, terapeutas, directivos y por supuesto para los deportistas tanto a nivel internacional como nacional (Arias-Moreno et al., 2018).

En este orden es importante considerar que las cargas en el entrenamiento, las metodologías utilizadas, unido a las altas exigencias en los niveles de competitividad a las cuales con frecuencia se exponen los deportistas en las diferentes etapas y deportes, hace que sea necesario reflexionar sobre aspectos relacionados con la optimización en el entrenamiento deportivo y cómo prevenir las posibles lesiones que se pueden presentar por una incorrecta praxis o simplemente por sobre carga durante el entrenamiento (Sánchez et al., 2020; Betancourt, Beatriz, Arias \& Barroso, 2020).

Los estudios precedentes a nivel internacional se han encaminado a resaltar la relación que se da entre las capacidades: fuerza-velocidad y sus impactos en la optimización del entrenamiento y en la prevención de lesiones. Sin embargo, alcanzar una visión más amplia y convincente de esta problemática y de cómo se ha estado abordando en el contexto de Latinoamérica y Ecuador sigue siendo un tema de particular interés, entre otros aspectos porque aún resultan escasos los estudios realizados en esta línea.

Complementando lo citado se destaca que como parte de la experiencia personal y durante el tiempo que se ha dedicado al proceso del entrenamiento deportivo, formando y entrenando deportistas se ha podido constatar mediante la observación realizada, que existen serias deficiencias en cuanto al empleo de metodologías adecuadas que permitan combinar el entrenamiento de la fuerza y la velocidad, como alternativa para garantizar el nivel óptimo en el entrenamiento. En muchas ocasiones el entrenamiento y la práctica se realizan sobre la base de conocimientos empíricos; esto impide como es lógico poder discernir qué trabajo o qué métodos son los más adecuados para aplicar en dependencia de los objetivos a lograr y de las características e individualidades de cada atleta y por 
consiguiente no se visualizan otras formas o herramientas de optimizar el entrenamiento (Capote, Rodríguez-Torres, Analuiza, Cáceres \& Rendón 2017), como es por ejemplo los métodos basado en la relación fuerza-velocidad $(\mathrm{F}-\mathrm{V})$.

Exclusivo interés requiere en este punto la prevención de lesiones, que como parte de praxis tradicionales o inadecuadas pueden ocurrir en cualquier momento del entrenamiento, trayendo consecuencias graves que incluso llegan a comprometer el desempeño deportivo del atleta. La experiencia de otros países ha ido demostrando que con un adecuado trabajo entre la relación F-V, se puede prevenir la ocurrencia de lesiones e incluso en el caso de existir una lesión la tendencia a la recuperación puede ser más rápida si se ha realizado el entrenamiento de ambas capacidades en igualdad de proporción. Varios autores señalan que un nivel más alto de fuerza está asociado a menor riesgo de lesiones, es decir, que es muy importante no solo para los deportistas, sino para la población en general entrenar y evaluar la fuerza.

Todo lo anterior en el contexto Latinoamericano y Ecuatoriano está asociado desde nuestra perspectiva con la necesidad de contar con literaturas que ayuden a clarificar con mayor profundidad este novedoso tema y sobre todo que expliquen cómo poder llevarlo a la práctica en los diferentes deportes. A ello se une la realización de programas de formación, capacitación y actualización para los entrenadores, de modo tal que les permitan evitar el empirismo durante el entrenamiento y estar actualizados en la introducción de nuevas metodologías que ayuden a tener una visión multidisciplinar del fenómeno objeto de estudio para poder indicar posibles soluciones. Cabe preguntarnos entonces: ¿Cómo influye la relación fuerza-velocidad en la optimización del entrenamiento y en la prevención de lesiones?

Dicha situación nos pone ante la necesidad de: Reflexionar sobre la relación entre la fuerza y la velocidad y su importancia para la optimización en el entrenamiento y la prevención de lesiones, declarándose este como el objetivo general de la investigación.

La novedad del tema y su alta significación en cuanto a las posibles transformaciones dentro de la metodología y praxis a realizar en el proceso de entrenamiento deportivo, aseguran su pertinencia y actualidad en el contexto de Latinoamérica con especial interés en Ecuador, de ahí la necesidad y justificación de la investigación que se presenta.

\section{Generalidades sobre las capacidades fuerza y velocidad.}

Se parte de considerar que tanto la fuerza como la velocidad son capacidades físicas determinantes en la práctica de cualquier disciplina deportiva.

Según Verhoshansky (2000), la fuerza es un componente fundamental para el rendimiento de todo ser humano y su correcto desarrollo. Apunta este autor que esta capacidad no puede ser olvidado en la preparación de los deportistas.

Mientras que la velocidad es entendida como: "la capacidad que permite un rendimiento óptimo al momento de realizar una acción deportiva, en el que la rapidez del movimiento 
es esencial ya que su realización efectiva dependerá de la velocidad con la que se realice" (Salmerón, 2017, p. 37).

Lo cierto es que ambas capacidades se ubican dentro de las capacidades condicionales y resultan fundamentales tanto en el desarrollo del ser humano como en la práctica deportiva. Al estar dentro del grupo de las capacidades condicionales requieren de ser desarrolladas y perfeccionadas para poder alcanzar buenos resultados deportivos.

\section{Relación entre fuerza-velocidad. Una teoría a sistematizar desde la visión de la optimización del entrenamiento y la prevención de lesiones.}

Para abordar este tema es necesario partir de dos interrogantes claves:

¿Existe o no relación entre estas dos capacidades?

¿Cómo influye la combinación de estas dos capacidades en la optimización del entrenamiento deportivo y en la prevención de lesiones?

En los últimos años las investigaciones aseveran la gran utilidad de establecer en la práctica deportiva en general la relación fuerza-velocidad, sin embargo, la mayoría de las investigaciones suelen analizar la fuerza y la velocidad como componentes aislados y no como una relación que tome lo mejor de cada una para la mejora del rendimiento deportivo y la prevención de lesiones.

Todo esto provoca cambios y transformaciones en las metodologías y formas de entrenamiento, al punto de aparecer un nuevo paradigma en el campo del entrenamiento deportivo. Dicho paradigma se centra en establecer la relación fuerza-velocidad (F-V), la misma que representa la mejor proporción entre las cualidades de fuerza y velocidad para extender los empujes balísticos, como los saltos, empujes, piques, etc. (Jiménez-Reyes et al., 2014).

En este sentido según Grosser (1992), la fuerza máxima, explosiva y velocidad componen una unidad dinámica. Debe señalarse que la relación (F-V) nos indica que, el aumento de la velocidad de acortamiento muscular va a disminuir la fuerza producida por el mismo y viceversa, esto quiere decir que está íntimamente relacionado con la potencia, y en el deporte lo importante es generar fuerza a altas velocidades. (Cross, Brughelli, Samozino, \& Morin, 2017; Morin et al., 2017). Según estudios de (Mendiguchia et al., 2016; Giroux et al., 2014; Suchomel et al., 2016), el entrenamiento deportivo basado en la relación F$\mathrm{V}$ ha ayudado a entrenadores a mejorar sus planificaciones, potenciar las capacidades físicas de sus atletas, reducir el impacto de test físicos invasivos como el de 1RM, a la evaluación de fuerza aplicada en diferentes músculos y articulaciones, a la prevención y rehabilitación de lesiones y como instrumento para comparar diferentes tipos de ejercicios.

Autores como Morin \& Samozino (2016), muestran en sus investigaciones como la relación F-V es utilizada para realizar un diagnóstico más individualizado y eficiente en 
ejercicios como el sprint y los saltos, en deportes como el atletismo, el rugby, el fútbol, el fútbol americano, el voleibol y el baloncesto donde es necesario producir una alta potencia mecánica.

Se comparte con los autores precedentes, quienes unidos a Bosch (2016), vienen desarrollando y aperturando con gran fortaleza la teoría de la relación fuerza-velocidad para contribuir a la optimización del entrenamiento deportivo. Explican la necesidad de establecer diferencias entre los ejercicios, según el vector de aplicación de la fuerza predominante

En base a la relación F-V se puede decir que, para el éxito de muchas actividades deportivas, es importante la habilidad de acelerar rápidamente el cuerpo desde una posición de reposo. Siguiendo el modelo mecánico y principio fisiológico muscular de Hill (1938), tal explosividad se relaciona directamente con las características mecánicas del componente contráctil del músculo y especialmente en la producción de potencia máxima.

Se ha afirmado que el rendimiento de múltiples ejercicios está determinado por la potencia máxima y la relación F-V del sistema neuromuscular involucrada en el movimiento ejecutado. Se necesitan pruebas confiables, válidas y sensibles para determinar estas importantes capacidades mecánicas de los músculos. Un método rápido y simple para determinar estos parámetros es a través de la evaluación de la relación F$\mathrm{V}$, obtenida de los movimientos de múltiples articulaciones cargadas. Se comparte con García-Ramos et al. (2016), al señalar que la manipulación de las condiciones de carga externa proporciona un amplio rango de valores de F y V (cargas pesadas: F alta y V baja; cargas ligeras: F baja y V alta).

\section{Del entrenamiento de la fuerza y la velocidad a la prevención de lesiones.}

El riesgo de lesiones, se encuentran presentes en la generalidad de las prácticas deportivas. Muchas están relacionadas con praxis inadecuadas, sobrecargas en los procesos de entrenamientos, diferenciación y ajuste a las posibilidades de cada atleta, o falta de actualización en la metodología a emplear. Como se conoce las lesiones son perjudiciales para los atletas, pueden causar una discapacidad o incluso terminar la carrera deportiva, también, son una carga para los clubes deportivos, ya que el tratamiento a menudo es exigente y costoso, impidiendo llegar a los resultados esperados.

Según el Instituto Nacional de Artritis y Enfermedades Musculoesqueléticas y de la Piel (NIH) de Estados Unidos, las lesiones deportivas son lesiones que ocurren durante la práctica de un deporte o al hacer ejercicio.

Declaran que existen dos tipos de lesiones deportivas:

- Las lesiones agudas ocurren de repente mientras se está jugando o haciendo ejercicio. 
- Las lesiones crónicas ocurren después de practicar un deporte o hacer ejercicio por mucho tiempo.

Un estudio realizado por Leppänen et al. (2014), resume los efectos del trabajo con la fuerza en las intervenciones y prevención de lesiones, se pudo observar la importancia de la fuerza para prevenirlas, demostrando que el entrenamiento de la fuerza es la mejor opción entre diferentes planes de ejercicios como los estiramientos, la propiocepción, multi-ejercicios entre otros, además, los efectos del entrenamiento de fuerza en lesiones de las extremidades inferiores se evaluaron en cuatro estudios con 1232 sujetos evaluados, y el entrenamiento de fuerza excéntrico redujo significativamente el riesgo de lesión en los isquiotibiales.

\section{Metodología.}

La metodología empleada en la presente investigación es de tipo descriptiva, no experimental con la utilización de métodos teóricos, dentro de estos se utilizaron: el histórico-lógico, el analítico - sintético, inductivo - deductivo y la revisión documental, los cuales permitieron revisar varias fuentes y bases de datos que contribuyeron a reflexionar sobre el problema objeto de estudio y su tratamiento en las diferentes bases de datos en un rango comprendido entre el 2014 y el 2020.

Dentro de las fuentes consultadas para obtener la información requerida sobre la relación fuerza-velocidad y su incidencia en la optimización del entrenamiento deportivo y la prevención de lesiones, se encuentran: Publice, Scopus, PubMed, Google Académico.

Para encontrar información adicional se realizó una búsqueda en los repositorios nacionales e internacionales que contemplan Tesis de Doctorado y Maestría. También se examinaron diferentes artículos publicados en otras bases y revistas como es la revista de Entrenamiento Deportivo y la revista de Educación Física, ambas recogidas en la base de datos Publice. Las palabras claves utilizadas para identificar los artículos y restringir la población en esta revisión fueron: fuerza, velocidad, entrenamiento deportivo, optimización y prevención de lesiones. Los dos primeros términos $(\mathrm{F}-\mathrm{V})$, fueron identificados con el conector AND, "force", "velocity", "profile", "relationship", "injury". Teniendo en cuenta la importancia de lograr la obtención de una información confiable y de calidad, la estrategia de búsqueda seguida permitió ir modificando la misma según cada base de datos consultada. Los términos fueron buscados según título, resumen y encabezamiento de materia.

\section{Criterios de inclusión}

1. Tipo de estudios: Estudios de revisiones teóricas o experimentales que sistematicen o desarrollen metodologías de entrenamiento que combinen la relación fuerza-velocidad en la optimización del entrenamiento deportivo y en la prevención de lesiones 
2. Tipo de participantes: deportistas practicantes de diferentes deportes, niveles y edades.

3. Tipo de resultados: Metodologías y Programas de entrenamiento que permitan demostrar la eficacia de la combinación y relación que se da entre la fuerza y la velocidad en la optimización del entrenamiento deportivo y en la prevención de lesiones

4. Tesis de Doctorado o Maestría realizadas en los tres últimos años en universidades Internacionales o Nacionales

\section{Criterios de exclusión}

1. Estudios o investigaciones realizadas en un rango de tiempo diferente al contemplado en la investigación y que no aporten elementos significativos para nuestro estudio.

2. Artículo en idioma diferente del español o inglés.

3. Otros estudios que por su tema o resultados no aporten elementos significativos para nuestra investigación.

\section{Evaluación de la calidad}

La calidad se determinó mediante el seguimiento riguroso de los criterios de inclusión y exclusión establecidos previamente.

Análisis de los resultados en cuanto a bases de datos consultadas:

- Resultados del análisis realizado en la base de datos Publice entre los años 2014 y 2020

Si bien es cierto que en esta base de datos se recogen estudios realizados desde el año 2011 hasta el 2020, para una cantidad de 318 artículos publicados, mucho de los cuales provienen de las revistas de Entrenamiento Deportivo y la de Educación Física; la consulta realizada determinó que entre los años 2014 y 2020 los estudios en torno al tema de investigación que nos ocupa se enmarcaron en dos líneas fundamentales: los relacionados con investigaciones sobre la fuerza y la potencia y otros dirigidos a evaluar los resultados del entrenamiento de la velocidad y la agilidad.

Comportándose de la siguiente forma:

Tabla No 1. Estudios sobre Fuerza y Potencia

\begin{tabular}{|c|c|c|c|c|c|c|c|}
\hline \multirow{2}{*}{$\begin{array}{ll}\text { Totalidad de } & \text { de } \\
\text { estudios } & \\
\text { publicados } & \end{array}$} & \multicolumn{7}{|c|}{ Totalidad de estudios realizados por año } \\
\hline & 2020 & 2019 & 2018* & 2017 & 2016 & 2015 & 2014 \\
\hline 110 & 17 & 18 & 21 & 17 & 18 & 3 & 16 \\
\hline
\end{tabular}


* Año con mayor prevalencia de publicaciones sobre este tema (2018)

Tabla No 2. Entrenamiento de la velocidad y la agilidad

\begin{tabular}{|c|c|c|c|c|c|c|c|}
\hline \multirow{2}{*}{$\begin{array}{l}\text { Totalidad de } \\
\text { estudios } \\
\text { publicados }\end{array}$} & \multicolumn{7}{|c|}{ Totalidad de estudios realizados por año } \\
\hline & 2020 & 2019 & 2018 & 2017 & 2016 & - 2015 & 2014 \\
\hline 15 & 1 & 1 & 3 & 1 & 3 & - & 7 \\
\hline
\end{tabular}

- Año con 0 , o menor número de publicaciones.

Como puede apreciarse tanto en la Tabla No. 1, como en la 2 se recogen aspectos importantes:

$\checkmark$ El número de investigaciones ha tenido un comportamiento desigual en ambos temas.

$\checkmark$ En relación con las investigaciones que abordan estudios sobre fuerza y potencia el año de mayor incidencia fue el 2018, mientras que los estudios sobre el entrenamiento de la velocidad y la agilidad mantiene igualdad de cantidad de investigaciones entre los años 2018 y 2016, resultando significativo el hecho de que en el 2015 no aparecen registros de investigaciones sobre este tema.

$\checkmark$ La otra particularidad observable es que existe mayor cantidad de publicaciones sobre los estudios de fuerza y potencia, mientras que la proporción de estudios sobre velocidad y agilidad es menor.

$\checkmark$ Resultando fundamental destacar que en la mayoría de las investigaciones realizadas se trabaja de manera independiente cada una de estas capacidades y no de forma combinada, lo cual constituye una limitación para nuestra investigación.

De todo lo señalado se deriva la necesidad de sistematizar las teorías en relación con la combinación del entrenamiento de la fuerza y la velocidad como una alternativa viable para optimizar el rendimiento deportivo y la prevención de lesiones.

Si debemos enfatizar que existieron 8 artículos publicados en esta base de datos en el rango de tiempo establecido para esta investigación, que cumplieron con los criterios de inclusión y exclusión establecidos por el investigador para realizar la búsqueda, los cuales aportaron elementos muy interesantes, facilitando fundamentar y demostrar los beneficios que en el orden de la optimización del entrenamiento deportivo y la prevención de lesiones tiene considerar como metodología de trabajo donde prime la combinación de la fuerza y la velocidad, indicando la necesidad de ajustar los procesos de intervención según características de los atletas participantes, tipo de deportes y edades.

Obsérvese en la Tabla No. 3, los principales aportes encontrados en los estudios referenciados y que se relacionan con el tema investigado.

Tabla No 3. Resultados de la búsqueda realizada en Publice (2014- 2020). Artículos referentes para la investigación. 


\begin{tabular}{|c|c|c|c|c|}
\hline Nombre del autor & Titulo & Año & $\begin{array}{l}\text { Base de publicación } \\
\text { y tema }\end{array}$ & Aportes \\
\hline $\begin{array}{l}\text { Joan Aguilera- } \\
\text { Castells }^{1} \text {, Bernat } \\
\text { Buscà }^{1} \text {, Jose } \\
\text { Morales }^{1} \text {, Mónica } \\
\text { Solana- } \\
\text { Tramunt }^{1} \text {, Azahara } \\
\text { Fort- } \\
\text { Vanmeerhaeghe, Ferna } \\
\text { ndo Rey- } \\
\text { Abella }{ }^{2} \text { Jaume } \\
\text { Bantulà }{ }^{1} \text { y Javier Peña }\end{array}$ & $\begin{array}{l}\text { Actividad } \\
\text { Muscular } \\
\text { Sentadilla } \\
\text { Búlgara. Efectos } \\
\text { Vibración } \\
\text { Adicional, } \\
\text { Suspensión y } \\
\text { Superficie } \\
\text { Inestable }\end{array}$ & 2020 & $\begin{array}{l}\text { Publice (Trabajo con } \\
\text { Fuerza) }\end{array}$ & $\begin{array}{l}\text { El estudio examinó el efecto de } \\
\text { los dispositivos de suspensión } \\
\text { (TRX®), las superficies } \\
\text { inestables (BOSUß) y las placas } \\
\text { de vibración sobre la actividad y } \\
\text { la fuerza muscular durante la } \\
\text { sentadilla búlgara }\end{array}$ \\
\hline Lic. Andrés Lépori & $\begin{array}{l}\text { Entrenamiento } \\
\text { de Fuerza en } \\
\text { Deportes } \\
\text { Acíclicos: ¿Qué, } \\
\text { ¿Cómo, } \\
\text { ¿Cuánto? }\end{array}$ & 2020 & $\begin{array}{l}\text { Publice (Trabajo con } \\
\text { Fuerza y Velocidad) }\end{array}$ & $\begin{array}{l}\text { Indica que cuando el objetivo } \\
\text { del entrenamiento de la fuerza es } \\
\text { optimizar el rendimiento en } \\
\text { acciones veloces (saltos, } \\
\text { aceleraciones, cambios de } \\
\text { dirección) y se utiliza la } \\
\text { sentadilla como uno de los } \\
\text { ejercicios para tal fin, las } \\
\text { intensidades más adecuadas se } \\
\text { encuentran en un rango } \\
\text { aproximado del } 60-80 \% \text { de } 1 \\
\text { RM y las pérdidas de velocidad } \\
\text { más beneficiosas entre el } 10 \text { y } \\
20 \% \text {. }\end{array}$ \\
\hline $\begin{array}{l}\text { Atle Hole } \\
\text { Saeterbakken }^{1} \text {, Vidar } \\
\text { Andersen }^{1} \text {, Roland Van } \\
\text { Den Tillaar }{ }^{2} \text {, Florian } \\
\text { Joly }^{3} \text {, Nicolay } \\
\text { Stien }^{1}, \text { Helene } \\
\text { Pedersen }^{1} \text {, Matthew } \\
\text { Peter Shaw }{ }^{1} \text { y Tom } \\
\text { Erik Jorung Solstad }\end{array}$ & $\begin{array}{l}\text { Los Efectos del } \\
\text { Entrenamiento } \\
\text { de la Fuerza de } \\
\text { Diez Semanas } \\
\text { sobre la Zona de } \\
\text { Estancamiento } \\
\text { en los Ejercicios } \\
\text { de Press de } \\
\text { Pecho }\end{array}$ & 2020 & $\begin{array}{l}\text { Publice (Trabajo con } \\
\text { Fuerza y Velocidad) }\end{array}$ & $\begin{array}{l}\text { Diez semanas con entrenamiento } \\
\text { de press de pecho incidieron en } \\
\text { la mejora de la fuerza, } \\
\text { disminuyendo la velocidad del } \\
\text { levantamiento y un aumento del } \\
\text { tiempo en cada fase de } \\
\text { levantamiento. Los autores } \\
\text { recomiendan la velocidad de } \\
\text { levantamiento en ejercicios de } \\
\text { press de pecho como una } \\
\text { medida importante al examinar } \\
\text { los levantamientos de repetición } \\
\text { máxima. }\end{array}$ \\
\hline Pablo Griboff & $\begin{array}{l}\text { Entrenamiento } \\
\text { de Sobrecarga } \\
\text { Excéntrica } \\
\text { Orientado al } \\
\text { Voleibol de } \\
\text { Alto } \\
\text { Rendimiento }\end{array}$ & 2020 & $\begin{array}{l}\text { Publice } 2020 \\
\text { (Prevención de } \\
\text { lesiones mediante } \\
\text { ejercicios } \\
\text { excéntricos) }\end{array}$ & $\begin{array}{l}\text { Desarrolla un análisis descriptivo } \\
\text { del ejercicio excéntrico como } \\
\text { una herramienta válida para } \\
\text { optimizar la performance y } \\
\text { prevenir lesiones en los } \\
\text { jugadores de voleibol, destacar } \\
\text { las adaptaciones que genera y } \\
\text { exponer estrategias de acción. } \\
\text { incluir activaciones excéntricas } \\
\text { en la preparación del voleibolista } \\
\text { ya que se ha comprobado su } \\
\text { efectividad tanto en la } \\
\text { rehabilitación de lesiones } \\
\text { músculo-tendinosas, como así } \\
\text { también en la búsqueda de } \\
\text { optimizar el rendimiento del } \\
\text { jugador }\end{array}$ \\
\hline Lic. Andrés Lépori & $\begin{array}{l}\text { Evaluación y } \\
\text { Entrenamiento }\end{array}$ & 2020 & & $\begin{array}{l}\text { Se describe uno de los } \\
\text { componentes que integra las }\end{array}$ \\
\hline
\end{tabular}




\begin{tabular}{|c|c|c|c|c|}
\hline & $\begin{array}{l}\text { de la Fuerza } \\
\text { como Medio } \\
\text { para la } \\
\text { Optimización } \\
\text { del Rendimiento } \\
\text { Neuromuscular } \\
\text { en Deportes } \\
\text { Acíclicos }\end{array}$ & & & $\begin{array}{l}\text { variables de prescripción del } \\
\text { entrenamiento de fuerza: tipo de } \\
\text { ejercicio, diferenciando sus } \\
\text { características cinéticas y } \\
\text { cinemáticas más importantes, } \\
\text { mientras que no se hace } \\
\text { referencia a los métodos de } \\
\text { entrenamiento (que también } \\
\text { forman parte de dichas variables }\end{array}$ \\
\hline $\begin{array}{l}\text { Souhail } \\
\text { Hermassi, Aloui } \\
\text { Ghaith, René } \\
\text { Schwesig, Roy J. } \\
\text { Shephard y Mohamed } \\
\text { Souhaiel Chelly }\end{array}$ & $\begin{array}{l}\text { Efectos del } \\
\text { Entrenamiento } \\
\text { de Fuerza de } \\
\text { Corto Plazo y el } \\
\text { Tapering en } \\
\text { Fuerza Máxima, } \\
\text { Potencia } \\
\text { Máxima, } \\
\text { Velocidad de } \\
\text { Lanzamiento del } \\
\text { Balón y } \\
\text { Rendimiento del } \\
\text { Sprint en } \\
\text { Jugadores de } \\
\text { Balonmano }\end{array}$ & 2019 & $\begin{array}{l}\text { Publice Revista de } \\
\text { Entrenamiento } \\
\text { Deportivo, Volumen } \\
\text { 33, Número } 4 \text { del } \\
\text { año } 2019 .\end{array}$ & $\begin{array}{l}\text { El propósito fue evaluar el } \\
\text { efecto del entrenamiento de } \\
\text { fuerza de corto plazo y de dos } \\
\text { semanas de tapering en el } \\
\text { rendimiento físico de los } \\
\text { jugadores de balonmano. Las } \\
\text { diez semanas de entrenamiento } \\
\text { de fuerza progresiva seguidas de } \\
\text { dos semanas de tapering fueron } \\
\text { una táctica general eficaz para } \\
\text { aumentar la potencia muscular, } \\
\text { el rendimiento de sprint y la } \\
\text { velocidad de lanzamiento del } \\
\text { balón en los jugadores de } \\
\text { balonmano. }\end{array}$ \\
\hline $\begin{array}{l}\text { Hyun-Jung Kim, Jin- } \\
\text { Hyuck Lee, Sung-Eun } \\
\text { Ahn, Min-Ji } \\
\text { Park y Dae-Hee Lee }\end{array}$ & $\begin{array}{l}\text { Influencia de la } \\
\text { Rotura del } \\
\text { Ligamento } \\
\text { Cruzado } \\
\text { Anterior en la } \\
\text { Fuerza } \\
\text { Muscular del } \\
\text { Muslo y la } \\
\text { Relación } \\
\text { Isquiotibiales- } \\
\text { Cuádriceps: Un } \\
\text { Meta-Análisis }\end{array}$ & 2018 & $\begin{array}{l}\text { Publice } 2018 \\
\text { (Prevención de } \\
\text { lesiones) }\end{array}$ & $\begin{array}{l}\text { Comparó la fuerza muscular } \\
\text { isocinética de los músculos } \\
\text { cuádriceps e isquiotibiales, y la } \\
\text { relación isquiotibiales- } \\
\text { cuádriceps, de los miembros } \\
\text { inferiores lesionados y no } \\
\text { lesionados de los pacientes con } \\
\text { rotura del LCA. Concluye } \\
\text { señalando que las disminuciones } \\
\text { fueron observadas en los } \\
\text { músculos cuádriceps e } \\
\text { isquiotibiales de los pacientes } \\
\text { con rotura del LCA, con la } \\
\text { disminución en la fuerza del } \\
\text { cuádriceps que era } 3 \text { veces } \\
\text { mayor. Estas reducciones } \\
\text { desiguales aumentan } \\
\text { ligeramente la relación } \\
\text { isquiotibiales-cuádriceps en } \\
\text { rodillas con lesión del LCA. }\end{array}$ \\
\hline $\begin{array}{l}\text { Andreas Bogdan } \\
\text { Anghel }\end{array}$ & $\begin{array}{l}\text { Optimización } \\
\text { del } \\
\text { entrenamiento } \\
\text { de fuerza para } \\
\text { mejorar el perfil } \\
\text { horizontal de } \\
\text { fuerza- } \\
\text { velocidad- } \\
\text { potencia en } \\
\text { velocistas de } \\
\text { alto rendimiento }\end{array}$ & 2017 & Publice 2017 & $\begin{array}{l}\text { El estudio está relacionado con } \\
\text { el atletismo de alto rendimiento } \\
\text { en la modalidad de carreras de } \\
\text { velocidad del Centro de Alto } \\
\text { Rendimiento Deportivo de } \\
\text { Madrid (CARD) establece una } \\
\text { propuesta de mejora, se elaboró } \\
\text { un marco teórico sobre } \\
\text { diferentes estudios y métodos } \\
\text { para mejorar el rendimiento en } \\
\text { la fuerza, el sprint y el perfil }\end{array}$ \\
\hline
\end{tabular}


ISSN: 2602-8085

\begin{tabular}{|l|l|l|l|}
\hline & & & \\
& & & \\
& & $\begin{array}{l}\text { horizontal de fuerza-velocidad- } \\
\text { potencia (F-V-P), así como } \\
\text { diferentes test de evaluación y } \\
\text { sus respectivas herramientas } \\
\text { tecnológicas. Finalmente, se } \\
\text { creó una guía con pautas para } \\
\text { optimizar el entrenamiento de } \\
\text { fuerza de los deportistas y } \\
\text { mejorar su perfil horizontal de } \\
\text { F-V-P. Esta guía, proporciona 4 } \\
\text { métodos para mejorar el perfil } \\
\text { horizontal de F-V-P de los } \\
\text { deportistas y por consecuencia, } \\
\text { su rendimiento deportivo. }\end{array}$ \\
\hline
\end{tabular}

Los datos mostrados en la Tabla No 3, reflejan que, de los 8 artículos seleccionados, 2 confirman la importancia del entrenamiento de la fuerza para la prevención de lesiones y el resto (6), enfatizan en la importancia de los ejercicios de fuerza y velocidad para garantizar la optimización del entrenamiento deportivo. Esto nos permite reafirmar la utilidad del tema de investigación y la significación que esto trae en los resultados deportivos. Implica por supuesto la necesidad de conocer, investigar y aplicar nuevas metodologías de entrenamiento, pero sin lugar a dudas, se comparte y se valora de positivo su utilización en el marco de los diferentes deportes.

Los estudios precedentes apuntan hacia el empleo de esta metodología y sistematizan las teorías para confirmar su utilidad. Según criterios del autor de la presente investigación es necesario el cambio, sustentado en la actualización e investigación sobre este novedoso tema y sus impactos en la optimización del entrenamiento deportivo y en la prevención de lesiones

- Resultados del análisis realizado en la base de datos Scopus entre los años 2014 y 2020

Para la revisión se tomaron 8 artículos comprendidos entre el 2014 y el 2020, publicados en base Scopus, los cuales guardan relación con el tema investigado y cumplen con los criterios de inclusión.

\section{Artículo No 1}

\begin{tabular}{|l|l|}
\hline $\begin{array}{l}\text { Año de } \\
\text { publicación }\end{array}$ & 2014 \\
\hline Título & $\begin{array}{l}\text { Effects of Resisted Sprint Training on Acceleration With Three Different Loads Accounting for } \\
5,12.5, \text { and 20\% of Body Mass }\end{array}$ \\
\hline Autores & Beatriz Bachero Mena, Juan José González Badillo \\
\hline Fuente: & $\begin{array}{l}\text { Journal of strength and conditioning research: the research journal of the NSCA, ISSN 1064- } \\
8011, \text { Vol. 28, N }{ }^{\circ} \text { 10, 2014, págs. 2954-2960 }\end{array}$ \\
\hline Aportes & $\begin{array}{l}\text { Señala que una resistencia que reduce la velocidad del atleta en más de un 10\% de sprint } \\
\text { descargado implicaría cambios sustanciales en la mecánica de sprint del atleta. }\end{array}$ \\
\hline
\end{tabular}

\section{Artículo No 2}

\begin{tabular}{|l|l|}
\hline $\begin{array}{l}\text { Año de } \\
\text { publicación }\end{array}$ & 2015 \\
\hline
\end{tabular}




\begin{tabular}{|l|l|}
\hline Título & Effects of Velocity-Based Resistance Training on Young Soccer Players of Different Ages \\
\hline Autores & $\begin{array}{l}\text { González Badillo, Juan J.; Pareja Blanco, Fernando; Rodríguez Rosell, David ; Abad Herencia, } \\
\text { José L.; del Ojo López, Juan J. ; Sánchez Medina, Luis }\end{array}$ \\
\hline Fuente: & $\begin{array}{l}\text { Journal of strength and conditioning research: the research journal of the NSCA, ISSN 1064- } \\
8011, \text { Vol. 29, No } 5,2015, \text { págs. 1329-1338 }\end{array}$ \\
\hline Aportes & $\begin{array}{l}\text { Analiza el efecto del entrenamiento de resistencia basado en la velocidad (RT) con cargas } \\
\text { moderadas y pocas repeticiones por conjunto combinadas con saltos y sprints en el rendimiento } \\
\text { físico, mostrando resultados favorables y diferencias significativas. }\end{array}$ \\
\hline
\end{tabular}

\section{Artículo No 3.}

\begin{tabular}{|l|l|}
\hline $\begin{array}{l}\text { Año } \\
\text { publicación }\end{array}$ & 2015 \\
\hline Título & Core stability. Concepto y aportaciones al entrenamiento y la prevención de lesiones \\
\hline Autores & $\begin{array}{l}\text { F.J. Vera-García, D. Barbado, V. Moreno-Pérez, S. Hernández-Sánchez y C. Juan-Recio, J.L.L. } \\
\text { Elvira }\end{array}$ \\
\hline Fuente & $\begin{array}{l}\text { Revista Andaluza de Medicina del Deporte } \\
\text { versión On-line ISSN 2172-5063versión impresa ISSN 1888-7546 Rev Andal } \\
\text { MedDeporte vol.8 no.2 Sevilla jun. 2015.http://dx.doi.org/10.1016/j.ramd.2014.02.004 }\end{array}$ \\
\hline Aportes & $\begin{array}{l}\text { Aporta una revisión de la literatura científica sobre la estabilidad de la zona central del } \\
\text { cuerpo (core stability) a pesar de que los ejercicios de core stability son elementos habituales } \\
\text { dentro de los programas de entrenamiento deportivo, no existen evidencias suficientes para } \\
\text { establecer una relación clara entre la práctica de estos ejercicios y la mejora del rendimiento en } \\
\text { el deporte. }\end{array}$ \\
\hline
\end{tabular}

\section{Artículo No 4.}

\begin{tabular}{|c|c|}
\hline $\begin{array}{l}\text { Año de } \\
\text { publicación }\end{array}$ & 2016 \\
\hline Título & Iniciación al entrenamiento de fuerza en edades tempranas: revisión \\
\hline Autores & G. Peña' J.R. Heredia' C. Lloret' M. Martín y M.E. Da Silva-Grigoletto \\
\hline Fuente: & $\begin{array}{l}\text { Revista Andaluza de Medicina del Deporte versión On-line ISSN 2172- } \\
\text { 5063versión impresa ISSN 1888-7546 Rev Andal Med } \\
\text { Deporte vol.9 no.1 Sevilla mar. 2016.http://dx.doi.org/10.1016/j.ramd.2015.01.022 }\end{array}$ \\
\hline Aportes & $\begin{array}{l}\text { Realiza una revisión sobre el entrenamiento de la fuerza en edades tempranas. Destacando los } \\
\text { beneficios del entrenamiento en edades tempranas siempre que esté organizado por técnicos y } \\
\text { especialistas capacitados y acorde a las particularidades e individualidades de los deportistas, } \\
\text { contemplando la dosificación adecuada de ejercicios neuromusculares y la planificación } \\
\text { progresiva de estos. }\end{array}$ \\
\hline
\end{tabular}

\section{Artículo No 5.}

\begin{tabular}{|l|l|}
\hline $\begin{array}{l}\text { Año } \\
\text { publicación }\end{array}$ & 2016 \\
\hline Título & $\begin{array}{l}\text { Entrenamiento combinado de fuerza y ejercicios de saltos, efectos sobre el rendimiento en el } \\
\text { salto vertical en un grupo de alto nivel de jugadores de voleibol durante una temporada } \\
\text { completa de competición }\end{array}$ \\
\hline Autores & Carlos García Asencio; Miguel Sánchez Moreno ; Juan José González Badillo \\
\hline Fuente: & $\begin{array}{l}\text { Retos: nuevas tendencias en educación física, deporte y recreación, ISSN 1579-1726, No. 29, } \\
\text { 2016, págs. 140-143 }\end{array}$ \\
\hline Aportes & $\begin{array}{l}\text { El estudio examina los efectos del entrenamiento combinado de fuerza y ejercicios de saltos sobre } \\
\text { el rendimiento en el SV, en un grupo de alto nivel de jugadores de voleibol durante una temporada } \\
\text { completa de la competición. Los resultados sugieren que el uso de cargas moderadas podría } \\
\text { producir resultados similares en el rendimiento del SV al uso de cargas elevadas, indican además } \\
\text { que la especificidad del entrenamiento, expresada por la proximidad de las velocidades de } \\
\text { ejecución de los ejercicios de entrenamiento a la velocidad de ejecución del SV, es determinante } \\
\text { para el rendimiento. }\end{array}$ \\
\hline
\end{tabular}

Artículo No 6. 


\begin{tabular}{|l|l|}
\hline $\begin{array}{l}\text { Año de } \\
\text { publicación }\end{array}$ & 2018 \\
\hline Título & El papel del control de la velocidad de ejecución en el entrenamiento de fuerza \\
\hline Autores & Juan José González Badillo \\
\hline Fuente: & $\begin{array}{l}\text { Entrenamiento de fuerza y acondicionamiento: Journal NSCA Spain, ISSN-e 2445-2890, No.9, } \\
\text { 2018, págs. 8-11 }\end{array}$ \\
\hline Aportes & $\begin{array}{l}\text { Destaca que el único objetivo posible en el entrenamiento de fuerza es mejorar la velocidad ante } \\
\text { cualquier carga, señalando que es equivalente a mejorar la fuerza máxima aplicada ante cualquier } \\
\text { carga. }\end{array}$ \\
\hline
\end{tabular}

\section{Artículo No 7}

\begin{tabular}{|l|l|}
\hline $\begin{array}{l}\text { Año } \\
\text { publicación }\end{array}$ & 2019 \\
\hline Título & $\begin{array}{l}\text { Tiempo de recuperación después del ejercicio de fuerza con diferentes magnitudes de carga y } \\
\text { pérdida de velocidad en la serie }\end{array}$ \\
\hline Autores & $\begin{array}{l}\text { Fernando Pareja Blanco; Antonio Villalba Fernández Pedro J. Cornejo-Daza; Juan Sánchez- } \\
\text { Valdepeñas; Juan José González-Badillo }\end{array}$ \\
\hline Fuente: & $\begin{array}{l}\text { RED: Revista de entrenamiento deportivo = Journal of Sports Training, ISSN 1133-0619, Tomo } \\
33, \text { No. 2, 2019, págs. 21-30 }\end{array}$ \\
\hline Aportes & $\begin{array}{l}\text { Compara el tiempo de recuperación siguiendo cuatro protocolos diferentes de ejercicios de fuerza } \\
\text { en términos de magnitud de carga (60\% vs. 80\% 1RM - una repetición máxima) y pérdida de } \\
\text { velocidad en la serie (20\% vs. 40\%). }\end{array}$ \\
\hline
\end{tabular}

\section{Artículo No 8}

\begin{tabular}{|l|l|}
\hline $\begin{array}{l}\text { Año } \\
\text { publicación }\end{array}$ & 2020 \\
\hline Título & $\begin{array}{l}\text { Efecto de dos periodos de entrenamiento de fuerza sobre el rendimiento en los ejercicios de salto } \\
\text { vertical, tintorera y boost en natación sincronizada }\end{array}$ \\
\hline Autores & Francisco Ramón Escrivá-Sellés y Juan José González-Badillo \\
\hline Fuente: & $\begin{array}{l}\text { Apunts. Educación Física y Deportes, 142, 35-45. https://doi.org/10.5672/apunts.2014- } \\
\text { 0983.es.(2020/4).142.05 }\end{array}$ \\
\hline Aportes & $\begin{array}{l}\text { Comprueba el efecto de dos periodos de preparación diferenciados por los medios de } \\
\text { entrenamiento de la fuerza (con/sin carga externa añadida-pesas), en deportistas de natación } \\
\text { sincronizada. Los resultados mostraron un mayor efecto del entrenamiento con carga externa } \\
\text { añadida sobre el rendimiento de las nadadoras en el CMJ, existiendo transferencia sobre las } \\
\text { acciones específicas, lo que probablemente lleve a la mejora del rendimiento competitivo. }\end{array}$ \\
\hline
\end{tabular}

El análisis de las investigaciones consultadas en esta base de datos, confirma la importancia del tema de investigación y contribuyen a fundamentar la teoría referente a la importancia de establecer la relación fuerza-velocidad en los procesos de entrenamiento como una alternativa para optimizar el rendimiento deportivo y prevenir las lesiones. Especial interés tienen los trabajos desarrollados por Badillo, en el (2015, 2016, 2018, 2020), quien es uno de los autores que más ha aportado en estos años a defender y sistematizar esta metodología, destacando la alta relación que se logra entre fuerza - velocidad y optimización en los entrenamientos y por consiguiente de los resultados deportivos. En este orden de ideas se coincide con Peña (2016), cuando señala los beneficios del entrenamiento de la fuerza en edades tempranas, siempre que esté organizado por técnicos y especialistas capacitados y acorde a las particularidades e individualidades de los deportistas, teniendo en cuenta la dosificación adecuada de ejercicios neuromusculares y la planificación progresiva de estos. 
Otros autores como García et al. (2016), también sugieren que el uso de cargas moderadas podría producir resultados similares en el rendimiento del SV al uso de cargas elevadas, indican además que la especificidad del entrenamiento, expresada por la proximidad de las velocidades de ejecución de los ejercicios de entrenamiento a la velocidad de ejecución del SV, es determinante para el rendimiento. Todo esto contribuye a fundamentar el tema de investigación.

\section{Resultados del análisis realizado sobre Tesis de Doctorado y Maestría 2014 y 2020}

Varias son las investigaciones que desde el componente de formación Doctoral o Magister, se plantean este tema de investigación. Siguiendo los criterios de inclusión se determinan como potencialmente relevantes para el estudio 3, Tesis de Doctorado, realizadas en universidades extranjeras y 13 Tesis de Maestría desarrolladas en los dos últimos años en Universidades ecuatorianas, las cuales constituyen referente de los estudios precedentes que permiten apoyar la reflexión en torno a la problemática abordada

Dentro de las tesis de Doctorado consultadas se encuentran las siguientes:

> Bachero, B. Importancia de la fuerza muscular sobre el rendimiento físico y deportivo en atletas especialistas de 800 metros de alto nivel Tesis doctoral. Universidad Pablo de Olavide (2017).

La investigadora cita que en su estudio evalúa los efectos de un entrenamiento de sprint de 7 semanas, 14 sesiones, resistente al trineo en la aceleración con 3 cargas diferentes según un \% de masa corporal (BM): carga baja (LL: $5 \%$ BM, n a 7), carga media (ML: 12,5\% BM, n a 6), y alta carga (HL: 20\% BM, n a 6), en estudiantes jóvenes. Además, se analizaron los efectos en ejercicios no entrenados: salto de contramovimiento (CMJ), sentadilla de salto vertical cargada (JS) y sentadilla completa (SQ). Al seguir los 3 grupos el mismo programa de entrenamiento consistente en aceleraciones de sprint de esfuerzo máximo con las cargas respectivas asignadas. Los resultados logrados indican la existencia de diferencias significativas entre los grupos, quedando demostrada las mejoras provocadas productos del entrenamiento.

Sánchez Moreno, M. Factores determinantes del rendimiento en el ejercicio de dominadas y efectos del entrenamiento concurrente de fuerza y resistencia Tesis doctoral. Universidad Pablo de Olavide (2017).

Según cita el autor el estudio proporcionar información sobre la mejora de la metodología del entrenamiento del ejercicio de las dominadas, abordando de manera directa el conocimiento de dos elementos fundamentales para el propio sistema de entrenamiento. El primero de estos elementos es el conocimiento de la carga que se aplica al sujeto en dicho ejercicio, la cual será cuantificada y definida de manera precisa a través de la velocidad de la primera repetición (González-Badillo \& Sánchez-Medina, 2010). El segundo de estos elementos es la pérdida de velocidad en la serie (Sánchez-Medina \& 
González-Badillo, 2011), la cual, junto con el primer elemento, va definir el grado de esfuerzo al que el deportista es sometido (González-Badillo \& Ribas, 2002).

Esquina Carlos Otero "Efectos del entrenamiento de fuerza con distintas intensidades relativas y nivel de rendimiento inicial sobre las respuestas neuromuscular y hormonal" resistencia Tesis doctoral. Universidad Pablo de Olavide (2017).

Cita el autor que el control de la velocidad de ejecución durante el entrenamiento de fuerza ha dado lugar a un nuevo enfoque en el control y dosificación de la carga, el cual es denominado entrenamiento de fuerza basado en la velocidad o velocity based resistance training. La investigación se basa en esta metodología de entrenamiento, donde el control de la carga de entrenamiento se realiza de manera diaria en cada repetición a través de la velocidad de ejecución, tratando además de resolver una de las cuestiones planteadas previamente, como es el análisis del efecto producido por la aplicación de diferentes intensidades en el entrenamiento de fuerza. En las conclusiones del estudio el autor confirma que el 1 entrenamiento de fuerza con cargas bajas (40 - 60\% de 1RM) ofrece similares, e incluso mayores mejoras sobre las variables relacionadas con la fuerza en el ejercicio de sentadilla completa en comparación con el entrenamiento con cargas altas $(70-90 \%$ de $1 \mathrm{RM})$ realizando el mismo número de repeticiones totales en el ciclo de entrenamiento. La aplicación de un programa de entrenamiento con cargas bajas ofrece mejoras superiores tanto en el salto vertical como en la capacidad de sprint, principalmente en T10-20, T20 y T30.

Como puede notarse en las tres tesis de Doctorado, queda confirmado la importancia de aplicar metodologías de entrenamientos basada en a la combinación de la fuerza y la velocidad como una herramienta factible para alcanzar la optimización dentro del entrenamiento y mejorar los resultados deportivos, lo cual tiene correspondencia con nuestro problema e idea de investigación.

En cuanto a las tesis de Maestría consultadas se toman de referencia las realizadas en los últimos dos años en Ecuador en los programas de Maestría de Entrenamiento Deportivo de la Universidad de las Fuerzas Armadas (ESPE) y de la Universidad Estatal de Milagro (UNEMI). Al aplicar los criterios de selección se determina como referentes potenciales 8 tesis del Programa de Maestría de la Universidad de las Fuerzas Armadas (ESPE) y 5 de la Universidad Estatal de Milagro (UNEMI), para un total de 13 Tesis de Maestría consultadas.

Derivado del análisis realizado sobre las tesis de Maestría se establecen las consideraciones siguientes:

$\checkmark$ Resulta positivo los avances logrados en cuanto a investigaciones científicas en el contexto nacional en relación con la aplicación de ejercicios de fuerza para mejorar el rendimiento deportivo en los diferentes deportes, sin embargo, aún no 
se alcanza los niveles deseados y necesarios sobre todo en relación con su aplicación en edades tempranas.

$\checkmark$ En la mayoría de las investigaciones realizadas se evidencian resultados al trabajar aisladamente las capacidades fuerza y velocidad, tanto para la optimización en el entrenamiento como para la prevención de lesiones, siendo limitadas las investigaciones que combinen el trabajo con ambas capacidades.

$\checkmark$ El otro elemento a considerar y a nuestro criterio fundamental es la necesidad de ampliar las investigaciones que demuestren los beneficios y utilidad que trae consigo la combinación del trabajo de las capacidades fuerza-velocidad para la optimización del entrenamiento deportivo y para la prevención de lesiones.

Lo señalado indica además las limitaciones que existen en el contexto nacional en relación con el abordaje teórico - práctico sobre esta novedosa metodología, considerándose un aspecto esencial para perfeccionar los procesos actuales de entrenamiento deportivo y facilitar posesionarnos en mejores resultados en los diferentes deportes.

Como es lógico lograr alcanzar un cambio de paradigmas en este orden implica la necesidad de preparar y capacitar a los entrenadores, directivos y deportistas en formas y metodologías nuevas que permitan la optimización en el entrenamiento deportivo, sin que esto conlleve a lesiones por praxis inadecuadas.

Resultados del análisis realizado sobre otros artículos e investigaciones 2014 y 2020

Se consideró 1 artículo más como relevante, realizado por Pardos, Sagarra, Valarezo, Sandoval ML y Contreras (2017) y publicado en la Revista Cubana de Investigaciones Biomédicas, en el cual los autores sistematizan la teoría referida a la importancia de la aplicación de un programa de entrenamiento pliométrico como el más utilizado para mejorar el rendimiento de los jóvenes tenistas, destacando dentro de sus bondades el hecho de que se caracteriza por ser un programa de corta duración, barato y de fácil cumplimentación para entrenadores y preparadores físicos de tenis.

\section{Resumen de la búsqueda}

$\checkmark$ Es importante significar que en la búsqueda realizada entre los años 2014 y 2020 se pudo constatar que los estudios publicados en la base Publice, relacionados con el tema de investigación que nos ocupa se detectan 125 artículos, los cuales se enmarcaron en dos líneas fundamentales: los relacionados con investigaciones sobre la fuerza y la potencia y otros dirigidos a evaluar los resultados del entrenamiento de la velocidad y la agilidad. Determinándose potenciales para nuestra investigación un total de 8 artículos, de estos 2 son referentes para la prevención de lesiones mediante el trabajo combinado entre las capacidades de fuerza y velocidad y el resto aborda la utilidad desde el contexto deportivo.

$\checkmark$ La particularidad de las investigaciones publicadas en esta base es que en la mayoría se trabaja de manera independiente cada una de estas capacidades y no de forma combinada, lo cual constituye una limitación para nuestra investigación.

$\checkmark$ En el caso de la base Scopus se determinan 8 investigaciones relevantes en donde queda confirmada la importancia de establecer la relación fuerza-velocidad en los 
procesos de entrenamiento como una alternativa para optimizar el rendimiento deportivo y prevenir las lesiones. Especial interés tienen los trabajos desarrollados por (Badillo, 2015, 2016, 2018, 2020; Peña y García, 2016; Morin \& Samozino, 2016), autores que sistematizan la teoría en relación con las metodologías donde se combinen ambas capacidades.

$\checkmark$ Se revisaron un total de 3 Tesis de Doctorado, realizadas en universidades extranjeras y 13 Tesis de Maestría desarrolladas en los dos últimos años en Universidades ecuatorianas, las cuales constituyen referente de los estudios precedentes que permiten apoyar la reflexión en torno a la problemática abordada. Destacándose en la generalidad de las tesis de doctorado la utilidad de combinar el trabajo con las capacidades fuerza - velocidad como parte del entrenamiento conducente a la mejora del rendimiento deportivo, sin embargo en el caso de las investigaciones de maestría aún son limitadas las investigaciones que combinen el trabajo con ambas capacidades, creando la necesidad de ampliar la visión en torno al desarrollo de nuevas líneas e investigaciones que permitan fundamentar y sistematizar el trabajo con estas nuevas metodologías dentro del entrenamiento deportivo en el contexto ecuatoriano.

$\checkmark$ Finalmente, la revisión concluye con los aportes de un artículo el cual enfatiza en la importancia del desarrollo desde edades tempranas de programas entrenamiento pliométrico como los más utilizado para mejorar el rendimiento en los jóvenes tenistas.

$\checkmark$ Derivado de todo el análisis realizado se concluye que el tema en cuestión es novedoso, fundamentalmente en el contexto Latinoamericano y ecuatoriano, implicando la necesidad de introducir metodologías actualizadas en los procesos de entrenamiento en los diferentes deportes. El hecho de poder combinar en el entrenamiento el trabajo con las capacidades fuerza-velocidad resulta extremadamente importante para optimizar el rendimiento en los deportes y garantizar la prevención de lesiones.

$\checkmark$ Pudiera considerarse una opción validad la realización de programas de capacitación conducentes a lograr mayor actualización por parte de los entes implicados (directivos, entrenadores y deportistas)

\section{Conclusiones.}

A modo de conclusión el autor de la presente investigación considera que:

- Existe una estrecha relación entre las capacidades fuerza-velocidad como fundamento para alcanzar la optimización en el entrenamiento y la prevención de lesiones, sin embargo, en el contexto Latinoamericano y ecuatoriano, constituye aun una teoría a sistematizar con mayor intencionalidad desde una visión multidisciplinar y participativa, justificándose así la necesidad, pertinencia y actualidad del tema de investigación.

- La introducción de esta novedosa metodología no solo va a posibilitar la optimización del entrenamiento y la prevención de lesiones, sino también permite revolucionar las tradicionales metodologías que se emplean en el entrenamiento deportivo, para migrar a metodologías más actualizadas que necesariamente van a conducir al perfeccionamiento y optimización tanto del entrenamiento como de los resultados a alcanzar en los diferentes niveles y deportes. 
- Será necesario pensar en el desarrollo de programas de capacitación y en la apertura de líneas de investigación que ayuden a sistematizar tanto de forma teórica como en la práctica las ideas en relación con la aplicación de estrategias y metodologías de entrenamiento que combinen el trabajo con la fuerza y la velocidad como una herramienta viable para lograr la optimización en el entrenamiento deportivo y la prevención de lesiones.

\section{Referencias Bibliográficas}

Arias-Moreno, E., Martínez, J., Goyes, F., Ortiz, V., \& Montero, S. (2018). Variabilidad en el rendimiento físico de las jugadoras de fútbol según las fases del ciclo menstrual. EmásF: Revista Digital de Educación Física, 51(51), 11-30.

Badillo, J. J. G. (2018). El papel del control de la velocidad de ejecución en el entrenamiento de fuerza. Entrenamiento de fuerza y acondicionamiento: Journal NSCA Spain, (9), 8-11.

González-Badillo, J., Pareja-Blanco, F., Rodríguez-Rosell, D., Abad-Herencia, J., OjoLópez, J. y Sánchez-Medina, L. (2015). Effects of Velocity-Based Resistance Training on Young Soccer Players of Different Ages. J Strength Cond Res, 29 (5), 1329-1338. Recuperado de https://doi.org/10.1519/JSC.0000000000000764

Bachero, B. \& Badillo, J. (2014). Effects of Resisted Sprint Training on Acceleration With Three Different Loads Accounting for 5, 12.5, and 20\% of Body Mass. Journal of strength and conditioning research: the research journal of the NSCA, 28 (10), 2954-2960. Recuperado de https://doi.org/ 10.1519/JSC.0000000000000492

Bachero, B. (2017). Importancia de la fuerza muscular sobre el rendimiento físico y deportivo en atletas especialistas de 800 metros de alto nivel. (Tesis doctoral). Universidad Pablo de Olavide, España.

Bentacourt, J. C., Sánchez, B., Arias, E., \& Barroso, E. (2020). Patrón de lateralidad en jugadores masculinos de baloncesto, reservas escolares y juveniles de La Habana. PODIUM-Revista de Ciencia y Tecnología en la Cultura Física, 15(3), 449-459.

Capote, G., Rodríguez-Torres, F., Analuiza, E., Cáceres, C., \& Rendón, P., (2017). El deporte, el entrenamiento deportivo y los entrenadores. Lecturas: Educación Física y Deportes, 22(234).

Cross, M., Brughelli, M., Samozino, P., \& Morin, J. (2017). Methods of Power ForceVelocity Profiling During Sprint Running: A Narrative Review. Sports Medicine, 47(7), 1255-1269. Recuperado de https://doi.org/10.1007/s40279-016-0653-3

Cross, M., Samozino, P., Brown, S., \& Morin, J. (2018). A comparison between the force-velocity relationships of unloaded and sled-resisted sprinting: single vs. multiple trial methods. European Journal of Applied Physiology, 118 (3), 563571. Recuperado de https://doi.org/10.1007/s00421-017-3796-5

Escrivá-Sellés, F. R. \& González-Badillo, J.J. (2020). Effect of Two Periods of Power Training on Performance in the Thrust, Barracuda and Boost Exercises in 
Synchronised Swimming. Apunts. Educación Física y Deportes, 142, 35-45. Recuperado de https://doi.org/10.5672/apunts.2014-0983.es.(2020/4).142.05

Esquina C., (2017). Efectos del entrenamiento de fuerza con distintas intensidades relativas y nivel de rendimiento inicial sobre las respuestas neuromuscular y hormonal resistencia. (Tesis doctoral). Universidad Pablo de Olavide, España.

García C,. Sánchez Moreno, M,. Badillo, J. (2016). Entrenamiento combinado de fuerza y ejercicios de saltos, efectos sobre el rendimiento en el salto vertical en un grupo de alto nivel de jugadores de voleibol durante una temporada completa de competición. Retos: nuevas tendencias en educación física, deporte y recreación, 20, 140-143.

García-Ramos, A., Jaric, S., Padial, P. \& Feriche, B. (2016). Force-velocity relationship of upper body muscles: Traditional versus ballistic bench press. Journal of Applied Biomechanics, 32(2), 178-185. Recuperado de https://doi.org/10.1123/jab.2015-0162

Giroux, C., Rabita, G., Chollet, D., \& Guilhem, G. (2014). What is the best method for assessing lower limb force-velocity relationship? International Journal of Sports Medicine, 36(2), 143-149. Recuperado de https://doi.org/10.1055/s-00341385886

Peña, G., Heredia, J., Lloret, C., Martín, M. y Da Silva-Grigoletto, M. (2016). Iniciación al entrenamiento de fuerza en edades tempranas: revisión. Revista de Revista Andaluza de Medicina del Deporte, 9 (1), 41-49. Recuperado de https://dx.doi.org/10.1016/j.ramd.2015.01.022

Grosser, M. (1992). Entrenamiento de la velocidad. Fundamentos, métodos y programas. Barcelona: Martínez Roca.

Haugen, T., Breitschädel, F., \& Seiler, S. (2019). Sprint mechanical variables in elite athletes: Are force-velocity profiles sport specific or individual? Plos one, 14(7). Recuperado de https://doi.org/10.1371/journal.pone.0215551

Helland, C., Haugen, T., Paulsen, G., Rakovic, E., Eriksrud, O., Seynnes, O., \& Mero, A. (2019). Force-velocity profiling of sprinting athletes: single-run vs. multiple-run methods. European Journal of Applied Physiology, 119(2). Recuperado de https://doi.org/10.1007/s00421-018-4045-2

Jiménez-Reyes, P., Samozino, P., Brughelli, M., \& Morin, J. (2017). Effectiveness of an individualized training based on force-velocity profiling during jumping. Journal Frontiers in Physiology, $7 . \quad$ Recuperado de https://doi.org/10.3389/fphys.2016.00677

Jiménez-Reyes,Samozino, P., Cuadrado, V., Conceição, F., González-Badillo, J. J., \& Morin, J. B. (2014). Effect of countermovement on power-force-velocity profile. European Journal of Applied Physiology, 114(11), 2281-2288. Recuperado de https://doi.org/10.1007/s00421-014-2947-1.

Lahti, J., Jiménez-Reyes, P., Cross, M. R., Samozino, P., Chassaing, P., Simond-Cote, B., Ahtiainen, J. P., \& Morin, J.-B. (2020). Individual Sprint Force-Velocity Profile Adaptations to In-Season Assisted and Resisted Velocity-Based Training in 
ISSN: 2602-8085

Vol. 5, N¹, p. 51-72, Enero - Marzo, 2021

Professional Rugby. Journal Sports. Recuperado de https://doi.org/10.3390/sports8050074.

Leppänen, M., Aaltonen, S., Parkkari, J., Heinonen, A., \& Kujala, U. M. (2014). Interventions to prevent sports related injuries: A systematic review and metaanalysis of randomised controlled trials. Sports Medicine, 44(4), 473-486. Recuperado de https://doi.org/10.1007/s40279-013-0136-8.

Mendiguchia, J., Edouard, P., Samozino, P., Brughelli, M., Cross, M., Ross, A., Gill, N., \& Morin, J. B. (2016). Field monitoring of sprinting power-force-velocity profile before, during and after hamstring injury: two case reports. Journal of Sports Sciences, 34(6), 535-541. Recuperado de https://doi.org/10.1080/02640414.2015.1122207.

Morin, J. B., Petrakos, G., Jiménez-Reyes, P., Brown, S. R., Samozino, P., \& Cross, M. R. (2017). Very-heavy sled training for improving horizontal-force output in soccer players. International Journal of Sports Physiology and Performance, 12(6), 840-844. Recuperado de https://doi.org/10.1123/ijspp.2016-0444

Morin, J. B., \& Samozino, P. (2016). Interpreting power-force-velocity profiles for individualized and specific training. International Journal of Sports Physiology and Performance, 11(2), 267-272. Recuperado de https://doi.org/10.1123/ijspp.2015-0638.

Pareja, F,. Blanco, A,. Villalba, P., Cornejo-Daza; Sánchez-Valdepeñas, J. y Badillo, J. (2019). Tiempo de recuperación después del ejercicio de fuerza con diferentes magnitudes de carga y pérdida de velocidad en la serie. RED: Revista de entrenamiento deportivo = Journal of Sports Training, 33 (2), 21-30.

Pardos, E., Sagarra, Valarezo E, Sandoval, M. y Contreras T, (2017) Programas de entrenamiento para mejorar el rendimiento en jóvenes tenistas: revisión sistemática. Revista Cubana de Investigaciones Biomédicas, 36 (3), 1-12.

Sánchez-Moreno, M. (2017). Factores determinantes del rendimiento en el ejercicio de dominadas y efectos del entrenamiento concurrente de fuerza y resistencia. (Tesis Doctoral). Universidad Pablo de Olavide, España.

Salmerón, M. (2017). Nuevas perspectivas sobre los factores determinantes del rendimiento en sprint: Perfil Fuerza-velocidad, entrenamiento y prevención de lesione. (Tesis Doctoral). Universidad Católica de Murcia, España.

Sánchez, B., Lastres, A., Arias, E. R., Mesa, M., Vidarrueta, R., \& García, L. D. (2020). Deportes de combate, hacia un modelo de finalidad táctica de selección de talentos. PODIUM-Revista de Ciencia y Tecnología en la Cultura Física, 15(3).

Suchomel Timothy J., Paul Comfort, and Jason P. Lake (2016). Enhancing the Force Velocity Profile of Athletes Using Weight- Lifting Derivatives. Strength and Conditioning Journal. Recuperado de https://doi.org/10.1519/SSC.0000000000000275.

Verhoshansky, Y. (2000). Superentrenamiento. Barcelona: Paidotribo.

Vera-García, F., Barbado, D., Moreno-Pérez, V., Hernández-Sánchez, S., Juan-Recio, C. y Elvira, J. (2015). Core stability. Concepto y aportaciones al entrenamiento y la 
prevención de lesiones. Revista Andaluza de Medicina del Deporte, 8 (2), 79-85. Recuperado de https://doi.org/10.1016/j.ramd.2014.02.004 
PARA CITAR EL ARTÍCULO INDEXADO.

Morocho Ubidia, C. R. (2021). LA RELACIÓN FUERZA-VELOCIDAD PARA LA OPTIMIZACIÓN DEL ENTRENAMIENTO Y PREVENCIÓN DE LESIONES. Ciencia Digital, 5(1), 51-72. https://doi.org/10.33262/cienciadigital.v5i1.1462

\section{¿Ciencia}

El artículo que se publica es de exclusiva responsabilidad de los autores y no necesariamente reflejan el pensamiento de la Revista Ciencia Digital.

El artículo queda en propiedad de la revista y, por tanto, su publicación parcial y/o total en otro medio tiene que ser autorizado por el director de la Revista Ciencia Digital.
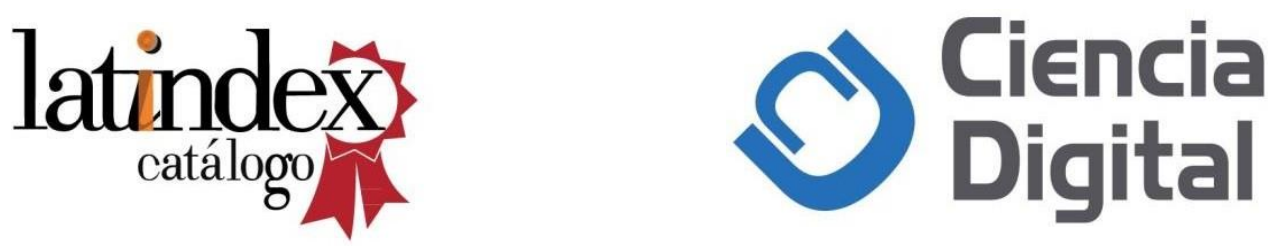